\title{
Norepinephrine Secretion in the Hypothalamic Paraventricular Nucleus of Rats during Unlimited Access to Self-Administered Nicotine: An In Vivo Microdialysis Study
}

\author{
Yitong Fu, Shannon G. Matta, Victoria G. Brower, and Burt M. Sharp \\ Department of Pharmacology, Health Science Center, University of Tennessee, Memphis, Tennessee 38163
}

Norepinephrine (NE) secretion within the hypothalamic paraventricular nucleus (PVN) is pivotal to endocrine and behavioral responses. Activation of NE afferents to PVN also is necessary for the hypothalamo-pituitary-adrenal axis response to passively administered nicotine. The mode of drug delivery is a critical determinant of the dynamics of neurotransmitter secretion, yet the PVN NE response to nicotine self-administration (SA) is unknown. Herein, rats housed in operant chambers had unlimited $23 \mathrm{hr}$ access to self-administered nicotine. In vivo microdialysis of PVN NE was performed, collecting consecutive $7 \mathrm{~min}$ samples over $9 \mathrm{hr}$ sessions during three phases of nicotine SA: acquisition (day 1); early maintenance, once stable rates of SA were achieved (day $9.2 \pm 0.6$ ); later maintenance (day $18.6 \pm 0.8$ ). On $\mathrm{d} 1$, nicotine animals had an increased percentage of SA episodes (SAEs) in which NE levels were elevated (80 vs $30 \%$ with saline; $p<0.01$ ). By early maintenance, a fourfold increase in such episodes was observed in nicotine animals $(p<0.01)$, and the overall NE level was greater ( $1.30 \pm 0.24$ vs $0.63 \pm 0.07 \mathrm{pg} / 10 \mu$ in saline; $p<0.05)$; $\mathrm{NE}$ increased during the first, but not the last, SAE. The pattern was similar during later maintenance, although NE responsiveness declined (overall NE level, $0.96 \pm 0.19$ in nicotine vs $0.52 \pm 0.08 \mathrm{pg} / 10 \mu \mathrm{l}$ in saline; $p<0.05$ ). Therefore, nicotine SAEs were associated with sustained increases in NE secretion during all three phases of SA. However, the reduced NE responsiveness observed both within the dialysis session in each phase and by later versus early maintenance is consistent with progression of partial daily desensitization of PVN NE secretion to nicotine SA. Therefore, in rats chronically self-administering nicotine, the drug stimulates sustained PVN NE secretion that may alter neuroendocrine and behavioral responses mediated by the PVN. Compared with studies of chronic human smokers, our nicotine SA model may reflect the CNS noradrenergic responses that occur during human cigarette smoking.

Key words: norepinephrine; hypothalamus; nicotine; self-administration; in vivo microdialysis; desensitization
Nicotine, the major psychoactive agent in tobacco, mediates a multiplicity of CNS responses by stimulating the release of neurotransmitters, including norepinephrine (NE), dopamine, and acetylcholine (Summers and Giacobini, 1995). We demonstrated that the noradrenergic system is sensitive to passive administration of nicotine, injected intravenously or directly into noradrenergic regions (Matta et al., 1995; Fu et al., 1997). Because NE plays an important role in the regulation of diverse CNS physiological responses, determining the effect of chronic exposure to nicotine on central NE secretion, especially via self-administration (SA), is essential for understanding the central actions of the drug.

$\mathrm{NE}$ is pivotal to numerous hypothalamic functions, including responses to stressors by the hypothalamo-pituitary-adrenal (HPA) axis and the regulation of food intake (Pacak et al., 1995; Wellman, 2000). Over 20 neuropeptides have been identified within paraventricular nucleus (PVN) neurons (Palkovits, 1988), and brainstem NE projections regulate many of these (Sawchenko and Swanson, 1982). For example, numerous stressors induce the NE-dependent release of corticotropin-releasing hormone, which stimulates ACTH secretion and adrenocortical glucocorticoid release (Pacak et al.,. 1995). Also, PVN noradren-

\footnotetext{
Received April 24, 2001; revised Aug. 22, 2001; accepted Aug. 27, 2001.

This work was supported by National Institute on Drug Abuse Grant 03977.

Correspondence should be addressed to Dr. Burt Sharp, Department of Pharmacology, Health Science Center, University of Tennessee, 874 Union Avenue, Memphis, TN 38163. E-mail: bsharp@utmem.edu.

Copyright (ㄷ) 2001 Society for Neuroscience $0270-6474 / 01 / 218979-11 \$ 15.00 / 0$
}

ergic input affects the prefeeding release of neuropeptide $\mathrm{Y}$ under food-restricted conditions (Yoshihara et al., 1996). Thus, NE is pivotal to the control of PVN neurons mediating endocrine and behavioral responses.

Nicotine stimulates NE secretion in the PVN (Sharp and Matta, 1993), which is necessary for activation of the HPA axis, because subsequent ACTH secretion can be blocked by both nicotinic receptor antagonists and NE depletion (Matta et al., 1990, 1993b). Although these studies have demonstrated the effects of investigator-initiated (i.e., passive) nicotine on $\mathrm{NE}$ secretion in the brainstem-PVN circuit, PVN NE response to nicotine SA is uncharacterized. From a wider perspective, the effects of nicotine SA on NE secretion anywhere in the brain are unknown.

The significance of this is underscored by evidence that other drugs of abuse activate CNS circuits in a differential manner when self-administered versus passively delivered. For example, significantly higher nucleus accumbens acetylcholine was measured in cocaine self-administering rats compared with controls (Mark et al., 1999). Therefore, the mode of administration is an important determinant of the dynamics of central neurotransmitter secretion, making it likely that nicotine self-administration will have a distinctive effect on PVN NE secretion.

Recently, we published a nicotine self-administration model in which rats have virtually unlimited SA access to intravenously administered nicotine, without previous shaping, conditioning or food deprivation, and in their home cage (Valentine et al., 1997), thereby exposing them to daily amounts of nicotine that, on a 
milligram per kilogram basis, are comparable with human smokers (Benowitz and Jacob, 1984). This model, which simulates basic elements of human cigarette smoking, may provide insight into the effects of nicotine on regulation of central NE secretion. In vivo $\mathrm{PVN}$ microdialysis was performed on rats during three stages of chronic nicotine SA: acquisition, early maintenance, and later maintenance. To our knowledge, this is the first report characterizing neurotransmitter secretion in animals with $23 \mathrm{hr}$ unlimited access to self-administered nicotine and the first observation of CNS NE secretion in rats self-administering any drug.

\section{MATERIALS AND METHODS}

Materials. (-)Nicotine hydrogen tartrate (all doses based on the free base) and norepinephrine hydrochloride were purchased from Sigma (St. Louis, MO). Sodium dihydrogen phosphate monohydrate, EDTA, acetonitrile, phosphoric acid (all from Fisher Scientific, Fair Lawn, NJ), 1-octanesulfonic acid sodium salt (J. T. Baker, Phillipsburg, NJ), and triethylamine (Aldrich, Milwaukee, WI) were used to prepare the mobile phase for HPLC with electrochemical detection. Concentric dialysis probes were constructed with cellulose fiber dialysis tubing obtained from Spectrum (Laguna Hills, CA) and silica tubing (outer diameter, 148 $\mu \mathrm{m}$; inner diameter, $73 \mu \mathrm{m}$ ) from Polymicron Technologies Inc. (Phoenix, AZ). Operant chambers, circuit boards, interface modules, and SA software were purchased from Coulbourn Instruments (Allentown, PA).

Nicotine self-administration. Self-administration was performed according to our previously published protocol with the following modifications (Valentine et al., 1997). Seven days after acclimation to a reverse light cycle and handling, male Lewis rats (Harlan Sprague Dawley, Indianapolis, IN), weighing 250-350 gm (3.5 months old at onset of the acquisition phase of SA), were anesthetized with xylazine-ketamine (13 and $87 \mathrm{mg} / \mathrm{kg}$, i.m., body weight, respectively; Parke-Davis, Morris Plains, NJ). Chronic guide cannulas (20 gauge) were stereotaxically implanted bilaterally into the PVN; atlas coordinates were as follows: anteroposterior, $-1.8 \mathrm{~mm}$; dorsoventral, $-7.5 \mathrm{~mm}$; mediolateral, \pm 0.2 $\mathrm{mm}$, from bregma with a flat skull (Paxinos and Watson, 1986). After $7 \mathrm{~d}$ of recovery, rats received jugular cannulas under xylazine-ketamine anesthesia and then were immediately placed into operant chambers located within individual sound- and light-attenuating environmental enclosures, where they resided for the duration of the study. The jugular line, exteriorized through a polyethylene button placed between the shoulder blades, was protected by a metal spring attached to the button and connected to a dual channel swivel located outside the environmental enclosure. The lines for the microdialysis probe (see below) also were channeled through this protective metal tether and exited outside the enclosure. Rats were allowed to recover for $3 \mathrm{~d}$, during which time they received progressively higher injections of heparinized saline (hourly injections from 100 to $200 \mathrm{U} / \mathrm{ml}$ in $50 \mu \mathrm{l}$ ) and a daily injection of the antibiotic Baytril $(7.6 \mathrm{mg} / \mathrm{kg}$ in $0.1 \mathrm{cc}$, i.v.; Bayer Corp., Shawnee Mission, KS). After recovery, rats were randomly assigned to treatment groups, and the jugular lines were filled with either $0.03 \mathrm{mg} / \mathrm{kg}$ nicotine in $200 \mathrm{U} / \mathrm{ml}$ heparinized saline $(50 \mu \mathrm{l}$ delivered over $0.81 \mathrm{sec}$ per $300 \mathrm{gm}$ body weight) or heparinized saline. The nicotine solution was prepared every $7-14 \mathrm{~d}$ and stored in a foil-wrapped glass bottle at $4^{\circ} \mathrm{C}$. Every day, the nicotine solution remaining in the syringe was discarded and the syringe was refilled.

Each environmental enclosure was equipped with its own ventilation fan and house light; the light was scheduled to turn off daily at exactly 12:30 P.M. and on again at 12:30 A.M. The interior operant chamber contained two levers positioned $5 \mathrm{~cm}$ above the floor and a green cue light $1 \mathrm{~cm}$ above each that signaled the availability of nicotine. One lever was randomly assigned as the active bar and would signal the computerdriven pump to deliver a $50 \mu \mathrm{l} / 0.81 \mathrm{sec}$ bolus injection of nicotine or saline when pressed by the rat; pressing the other bar had no consequence. Injections were followed by a 7 sec time-out, during which the green light above the active bar was not illuminated and nicotine or saline were unavailable. Rats were not shaped, conditioned, or food deprived in preparation for lever pressing and were allowed to acquire selfadministration behavior throughout the $23 \mathrm{hr}$.

Environmental enclosures, operant chambers, and syringe pumps were controlled by Coulbourn Habitest Universal LabLink interfaces and computers located in an adjacent room. Each morning, during the final hour of the lights-on cycle (i.e., at 11:30 A.M.), the clock for the computer program (Coulbourn L2t2) had to be manually reset, necessi- tating a short interruption in nicotine availability. To accommodate this, as well as to accomplish animal husbandry needs, measurements of body weight, and data downloading, all of the environmental enclosures were opened and the green cue lights were turned off to signal this interruption to the rats. Levers were not retracted, and lever press activity was not recorded. Exactly $1 \mathrm{hr}$ later (12:30 P.M.), house lights shut off, cue lights were illuminated, nicotine (or saline) was again available, and enclosure doors were shut. All procedures were conducted in accordance with the NIH Guidelines Concerning the Care and Use of Laboratory Animals and were approved by the Animal Care and Use Committee of the University of Tennessee.

In vivo microdialysis. The microdialysis procedure was performed as described previously (Fu et al., 1997) with concentric microdialysis probes (molecular weight cutoff, 13,000 Da; outer diameter, $235 \mu \mathrm{m} ; 2$ $\mathrm{mm}$ dialysis membrane) constructed in our laboratory. The recovery efficiency for NE was $6.4 \pm 0.6 \%(n=10)$. Twelve hours before a microdialysis session, heparin was removed from the intravenous solution to minimize intracranial hemorrhage during probe insertion. On the morning of microdialysis, $3 \mathrm{hr}$ before lights out, a probe was inserted into the guide cannula, connected to the dual channel swivel, and perfused at $1.4 \mu \mathrm{l} / \mathrm{min}$ with a solution of Krebs' Ringer's buffer (KRB). During the daily $1 \mathrm{hr}$ interval when nicotine was unavailable (animal husbandry and equipment maintenance period), five consecutive $7 \mathrm{~min}$ microdialysis samples were obtained. Immediately thereafter, at lights out, nicotine was again made available, and the collection of 7 min samples (into $1 \mu \mathrm{l}$ of 5\% perchloric acid) continued for $9 \mathrm{hr}$. This $9 \mathrm{hr}$ period permitted the characterization of PVN NE release during approximately three-fourths of an animal's active lever presses, all of which resulted in the delivery of nicotine injections (Valentine et al., 1997).

Pilot studies showed a reduced number of active lever presses in a fraction of the animals during an initial microdialysis session. This, however, stabilized during the second microdialysis session (active lever presses, $35 \pm 4$ during the second $9 \mathrm{hr}$ microdialysis session vs $35 \pm 3$ during the corresponding $9 \mathrm{hr}$ period on the previous nondialysis day; $n=12$ ). Therefore, a preliminary microdialysis session was always conducted without sample collection $48 \mathrm{hr}$ before the actual session. In all experiments performed during acquisition, early maintenance, and late maintenance, the same side of the PVN was microdialyzed twice, separated by $48 \mathrm{hr}$. Probe position was verified histologically (Fu et al., 1997); only data from animals with correct placement were analyzed.

The KRB dialysis solution used in these investigations contained 147 $\mathrm{mm} \mathrm{NaCl}, 4.0 \mathrm{~mm} \mathrm{KCl}$, and $3.4 \mathrm{mM} \mathrm{CaCl}_{2}(0.2 \mu \mathrm{m}$ filter sterilized and degassed) with $5 \mu \mathrm{M}$ nomifensine (NE reuptake blocker) (Schacht et al., 1982). It has been reported that the concentration of calcium in KRB microdialysate can affect striatal dopamine release in response to D2 receptor agonists, in that lower concentrations of calcium (1.2 versus 3.4 $\mathrm{mm}$ ) enhanced the potency of the agonists (Timmerman and Westerink, 1991). Therefore, in a pilot study, microdialysis was performed during the early maintenance phase to determine whether $1.8 \mathrm{~mm}$ calcium KRB would modify the NE response to nicotine SA. The calcium concentration did not affect NE secretion [e.g., incremental NE responses to a self-administration episode (SAE)] or any of the parameters of nicotine SA (e.g., total number of nicotine injections per $23 \mathrm{hr}$ ).

The animals in these investigations were housed in their operant chambers, apparently resulting in very low stress and in correspondingly low PVN NE levels. The levels in microdialysates from these animals were usually $\sim 20-25 \%$ of those obtained from animals moved from group housing in a vivarium to acute chambers within several hours. Therefore, $3.4 \mathrm{~mm}$ calcium and nomifensine were used in combination to permit reliable detection of basal PVN NE levels, which otherwise would be undetectable in $\sim 80 \%$ of animals.

HPLC with electrochemical detection. As reported previously (Fu et al., 1997, 1998), microdialysate samples (10 $\mu \mathrm{l})$ were automatically injected by a CMA 200 refrigerated autosampler (CMA Microdialysis, North Chelmsford, MA) onto a $150 \times 2 \mathrm{~mm}$ ODS C18 column (ESA Inc., Chelmsford, MA) connected to an ESA model 580 HPLC pump. The mobile phase, containing $80 \mathrm{~mm}$ sodium dihydrogen phosphate monohydrate, $2.0 \mathrm{~mm}$ 1-octanesulfonic acid sodium salt, $100 \mu \mathrm{l} / \mathrm{l}$ triethylamine, 5 nM EDTA, and $10 \%$ acetonitrile, pH 3.0, was perfused at $0.25 \mathrm{ml} / \mathrm{min}$. NE levels were determined using an ESA 5041 high-sensitivity analytical cell and an ESA Coulochem II 5200A electrochemical detector at a potential of $220 \mathrm{mV}$ with the current gain at $5 \mathrm{nA}$. Under these conditions, the limit of detection for NE was $150 \mathrm{fg}$. The sample-to-sample variation in 10 consecutive injections of NE standard (500 fg) was calculated to be $5.16 \pm 2.91 \%$ (mean $\pm \mathrm{SD}$ ). Representative NE micro- 
dialysate chromatograms have been published previously (Fu et al., 1997).

Experimental protocols. The first experiment was designed to characterize the NE secretory response to nicotine SA during the first day of the acquisition phase. The acquisition phase was defined as the interval from the first day nicotine was available for SA (day 1) until maintenance criteria were achieved (see below). The second series of experiments characterized the NE response to nicotine SA at early and later time intervals during the maintenance phase of SA. The criterion for maintenance was a mean value for active lever presses during 3 consecutive days of $\geq 40$ presses, with a variance $\leq 15 \%$. This criterion was applied to each animal, and only animals meeting this criterion were included in the analyses. In addition, active lever presses during the 3 consecutive days were significantly greater than inactive presses. Maintenance was achieved within $5.8 \pm 2.0 \mathrm{~d}$ (mean $\pm \mathrm{SD}$ ), and dialysis was conducted $3 \mathrm{~d}$ later. The right or left PVN was randomly selected for early maintenance microdialysis, and the contralateral PVN was dialyzed during later maintenance, $10-12 \mathrm{~d}$ thereafter.

Because the results reported herein show a reduction in the NE response to nicotine SA by the end of the $9 \mathrm{hr}$ microdialysis period, subsequent experiments were designed to further clarify this observation. First, to test whether the efficiency of the probe decreased during the $9 \mathrm{hr}$ microdialysis period, the in vitro $\mathrm{NE}$ recovery rate was measured in six microdialysis probes both before and after $9 \mathrm{hr}$ of in vivo microdialysis. Second, to determine whether the reduction in NE secretion was attributable to diminished responsiveness to nicotine, rats were given two consecutive injections of nicotine at the onset of lights out and then again $7 \mathrm{hr}$ later. This experiment was performed when rats were in the early maintenance phase of nicotine SA, a time when NE secretion showed reduced responsiveness to nicotine. Therefore, to ensure that elevations of PVN NE were readily detectable, two consecutive injections of nicotine, rather than one, were given via computer-generated signals $(0.03$ $\mathrm{mg} / \mathrm{kg}$ twice, separated by a $7 \mathrm{sec}$ delay). All other nicotine was delivered as usual in response to active lever presses. Third, to determine whether the reduced NE response to nicotine SA was attributable to the depletion of axonal NE stores, $1 \mathrm{hr}$ after the second pair of programmed nicotine injections, yohimbine (an $\alpha_{2}$ adrenergic antagonist; $6.0 \mathrm{mg} / \mathrm{kg}$, i.p.) (Sharp and Matta, 1993), was administered to the same animals. Last, to determine whether the reduction in NE responsiveness to the second set of computer-programmed nicotine injections reflected a diurnal influence, two groups of animals that received saline after pressing the active lever were each tested by receiving two consecutive computer-driven injections of nicotine at only one of the two time points discussed above $(0$ or $+7 \mathrm{hr})$.

Data analysis. To obtain sufficient sample for the detection of PVN $\mathrm{NE}$, the collection interval for each microdialysis samples was $7 \mathrm{~min}$. However, rats often pressed the active lever more than once within the 7 min collection interval, summating the NE release and frequently extending it into the next sample. Therefore, all active lever presses occurring within $7 \mathrm{~min}$ of each other defined a single SAE. The NE release (i.e., incremental NE response) associated with such an SAE was calculated as the difference between the average microdialysate NE level in the two SAE samples (obtained during and immediately after that SAE) and the average NE levels present in the two samples immediately preceding the SAE (baselines). An SAE associated with increased NE secretion (i.e., at least $10 \%$ above the baseline NE levels) was defined as an increasing SAE (iSAE). The value of $10 \%$ was selected to capture a wide range of SAE-associated changes in NE release while excluding those that were within the range of variation attributable to HPLC measurement [sample-to-sample variation of $5.16 \pm 2.9 \%$ (mean $\pm \mathrm{SD})]$. Finally, the overall mean level of microdialysate NE was calculated using the values of all of the samples collected during the $9 \mathrm{hr}$ session.

Data (mean \pm SE) were expressed as picograms of NE per $10 \mu \mathrm{l}$ of microdialysate. Data were analyzed using one-way or two-way ANOVA and post hoc testing with Fisher's least significant difference (SPSS 7.5; SPSS, Chicago, IL) or appropriate $t$ tests.

\section{RESULTS}

Figure 1 shows the location within the PVN of the membrane area of all microdialysis probes used in these investigations. Histological evaluation showed that the membrane areas were all situated within the PVN, and the upper aspect of the membrane was located within the thalamus. In the few experiments in which

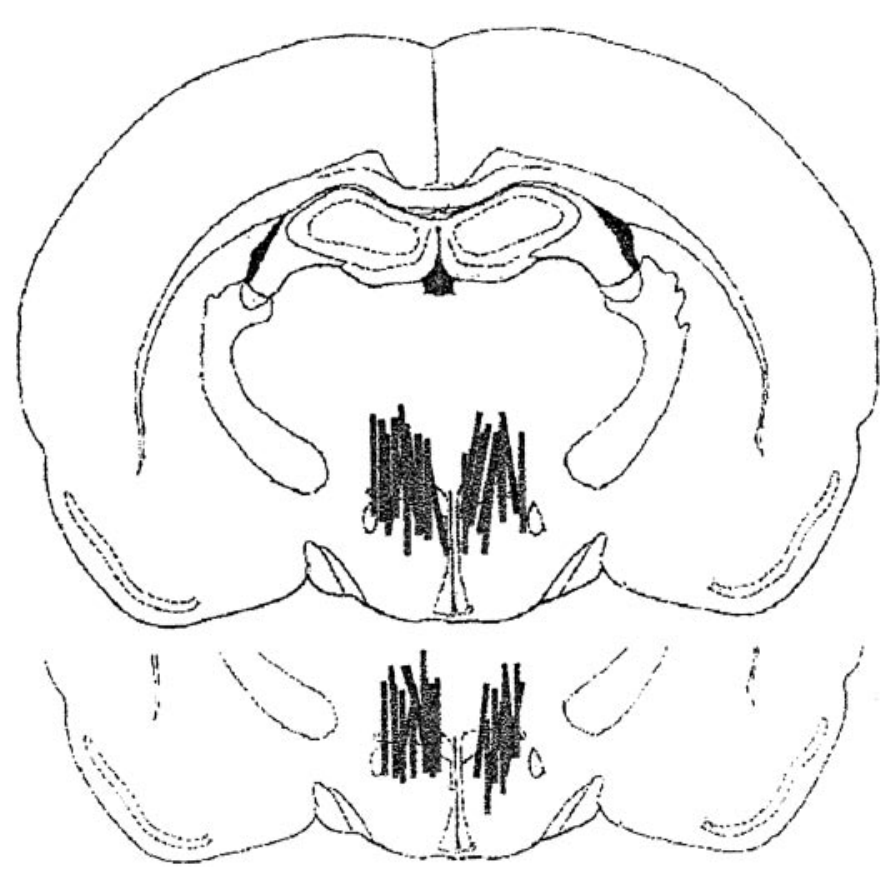

Figure 1. Diagrammatic representation of dialysis probe placement in the rat hypothalamic paraventricular nucleus. Histological analysis of Nissl-stained coronal sections showed the position of the membrane segment of each microdialysis probe used in all of the experiments. Each line segment illustrates the position of a dialysis membrane; two identical coronal sections are shown to accommodate all of the membranes (anteroposterior, $-2.1 \mathrm{~mm}$ ). Drawings are adapted from Paxinos and Watson (1986).

the tip of the probe was located in the thalamus (data not shown), $\mathrm{NE}$ was undetectable in the microdialysate.

\section{PVN NE secretion and SAEs during the acquisition phase}

Figure $2 A$ shows microdialysate NE levels in the PVN of a representative rat self-administering nicotine on the first day the drug was made available. Because the animal was acquiring the association between active lever pressing and receiving nicotine, the active lever was pressed only six times. The first four active lever presses occurred in very close succession, thereby constituting a single SAE (because lever presses were not recognized during the $7 \mathrm{sec}$ time-out after an active lever press, each active lever press shown resulted in a single programmed injection of nicotine). Two additional SAEs, at 344 and $403 \mathrm{~min}$, took place during the $9 \mathrm{hr}$ microdialysis session. The first SAE, at $3 \mathrm{~min}$, resulted in a large increase in NE secretion (iSAE, inverted triangle with $d o t$ ), and the other two iSAEs were accompanied by small NE responses ( $>10 \%$ of baseline). In comparison, an animal receiving saline (Fig. $2 B$ ) had five SAEs, only one of which (at $198 \mathrm{~min}$ ) was associated with a small increase in $\mathrm{NE}$ secretion and could be designated as an iSAE.

In Figure 3, the top two panels show the relationship of SAEs to $\mathrm{NE}$ release on day 1 of acquisition in rats receiving nicotine $(n=$ $6)$ versus saline $(n=6)$ when the active lever was pressed. $A 1$ shows that nicotine produced a doubling in the mean number of SAEs associated with increasing NE levels (iSAEs; $t=3.99 ; p<$ 0.01). $A 2$ shows that, compared with saline, nicotine increased the fraction of total SAEs that were accompanied by increasing $\mathrm{NE}$ levels (iSAE/total SAEs) from $\sim 30$ to $80 \%(t=7.58 ; p<0.01)$, without affecting the number of SAEs (Table 1). This demon- 
Figure 2. NE levels in PVN microdialysates obtained during day 1 of the acquisition period of selfadministration. NE levels (picograms per $10 \mu \mathrm{l}$ ), in dialysis samples collected every 7 min for $9 \mathrm{hr}$, are shown for two representative rats acquiring selfadministration behavior with $23 \mathrm{hr}$ unlimited access. Rats self-administered a bolus injection of nicotine ( $A ; 0.03 \mathrm{mg} / \mathrm{kg}$ body weight in $50 \mu \mathrm{l}$ of heparinized saline over $0.81 \mathrm{sec}$ ) or heparinized saline $(B)$ after pressing the active lever in the operant chamber. The filled circles indicate dialysis samples collected during the brief daily interruption in availability of nicotine or saline (for animal husbandry and resetting the computer clock); the open circles indicate consecutive samples taken once nicotine became available after lights out $(0 \mathrm{~min}) .+$ marks the time when the active lever was pressed to deliver an injection. The inverted triangles, indicating an SAE, point to the first of two consecutive samples used to calculate the NE response to lever presses (see Materials and Methods, Data analysis). The capped triangles indicate an SAE associated with NE levels at least $10 \%$ above baseline levels (i.e., an iSAE). In a rat acquiring nicotine SA, three of three SAEs were iSAEs compared with only one of five in a rat receiving saline in response to a lever press.

strates that, on the day drug SA was first initiated, animals receiving nicotine had an increased number of iSAEs.

Additional analyses were done to evaluate the magnitude of NE release associated with SAEs during the first day of nicotine SA. Figure $4 A$ shows that the mean NE increment associated with all iSAEs in the $9 \mathrm{hr}$ session was more than threefold greater in rats self-administering nicotine than saline $(t=5.39 ; p<0.01)$. In addition, in nicotine-treated rats, the average NE increment during the very first iSAE also was greater $(t=2.69 ; p<0.025)$ (Fig. $4 B$ ). In contrast, the average NE increment of the last iSAE was not significantly different between these two groups $(t=1.38$; $p>0.05$ ) (Fig. 4C). In the animals receiving nicotine, comparison of the NE increments associated with the first versus the last iSAE (Fig. 4B,C) also showed that the first NE response was significantly higher than the last $(t=2.72 ; p<0.025)$. Figure $4 D$ showed that the overall mean NE levels, calculated from all of the samples collected during the $9 \mathrm{hr}$ microdialysis session, were similar in both the nicotine and saline groups $(0.60 \pm 0.36$ vs $0.64 \pm 0.26 \mathrm{pg} / 10 \mu \mathrm{l} ; t=0.57 ; p>0.05)$. Thus, receiving nicotine on the first day it was made available resulted in iSAEs associated with significantly greater incremental NE responses compared with animals receiving saline. These nicotine-induced NE increments were associated with the first, but not the final, iSAEs recorded during the microdialysis session. These findings suggest that the NE response to self-administered nicotine may decline with repeated dosing and/or prolonged access to the drug during the initial $9 \mathrm{hr}$ that it is available. It is important to note that on day 1 of acquisition, the rats are just beginning to acquire the association between lever pressing and receiving nicotine. Because this association is not well established on day 1 , the PVN $\mathrm{NE}$ response to nicotine is not necessarily characteristic of nicotine SA.
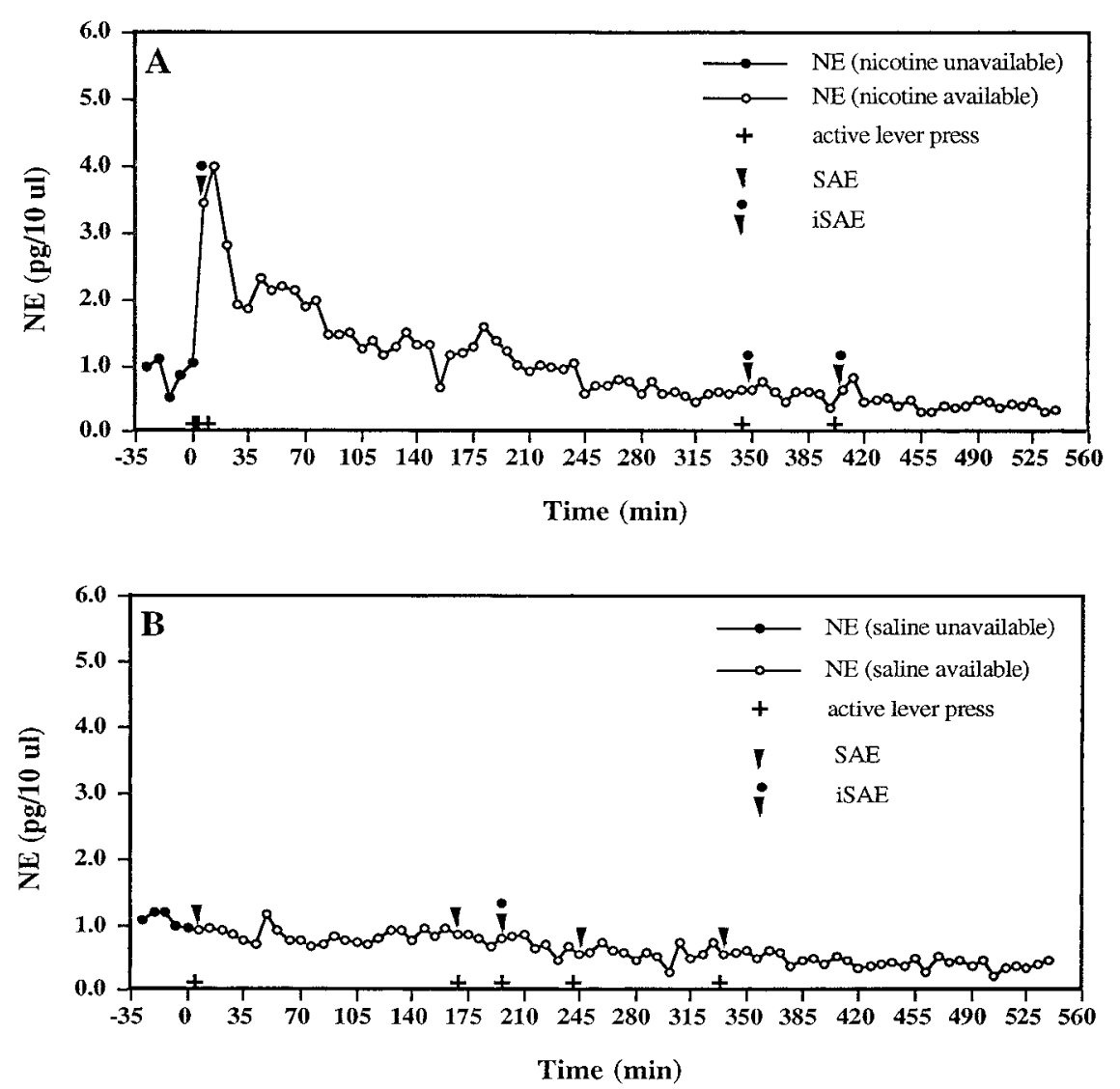

The numbers of active and inactive lever presses were compared during both the first $23 \mathrm{hr}$ SA session in which acquisition microdialysis was performed and the $3 \mathrm{~d}$ interval that defined the achievement of early maintenance. The following number of active versus inactive lever presses occurred within the first $23 \mathrm{hr}$ SA session during which microdialysis was performed: $30.3 \pm 13.4$ active lever presses versus $30.7 \pm 10.1$ inactive lever presses in the nicotine SA group $(t=0.03 ; p>0.05 ; n=6)$; and $22.0 \pm 4.3$ active lever presses versus $36.8 \pm 15.3$ inactive lever presses in the saline group ( $t=1.09 ; p>0.05 ; n=6)$. During the 3 consecutive days that defined the achievement of early maintenance, the number of active lever presses was twofold greater than inactive presses in rats self-administering nicotine (e.g., on the third consecutive day, active lever presses was $68.6 \pm 4.4$, and inactive lever presses was $34.4 \pm 8.6 ; F_{(2,102)}=12.06$ for two-way ANOVA of active vs inactive lever presses by 3 consecutive days, $p<0.01$; post hoc comparison of lever presses on the third consecutive day, $t=3.55, p<0.01)$. In contrast, no difference was found in active versus inactive lever presses in rats receiving saline (e.g., on the third consecutive day, active lever presses was $23.1 \pm 6.6$, and inactive lever presses was $\left.35.7 \pm 8.3 ; F_{(2,72)}=1.87, p>0.05\right]$.

\section{PVN NE secretion and SAEs during the early maintenance phase}

When microdialysis was performed in the early phase of maintenance, rats had achieved stable rates of SA and had been selfadministering nicotine for $9.2 \pm 0.6 \mathrm{~d}$ (range of 7-13 d). Figure 5, $A$ and $B$, shows SAEs and PVN NE secretion during $9 \mathrm{hr}$ dialysis sessions in two rats receiving nicotine $(n=12)$ versus saline $(n=$ 7), respectively. $A$ demonstrates that many iSAEs occurred throughout the session, whereas few iSAEs occurred in a saline rat $(B)$. In contrast to the acquisition phase, the early mainte- 


\section{Acquisition}
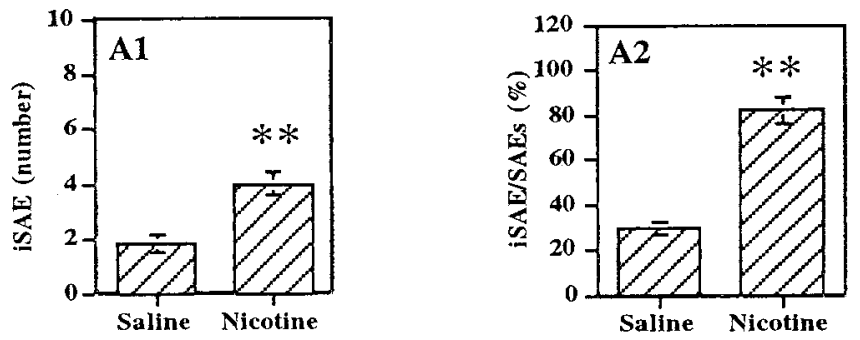

Early Maintenance
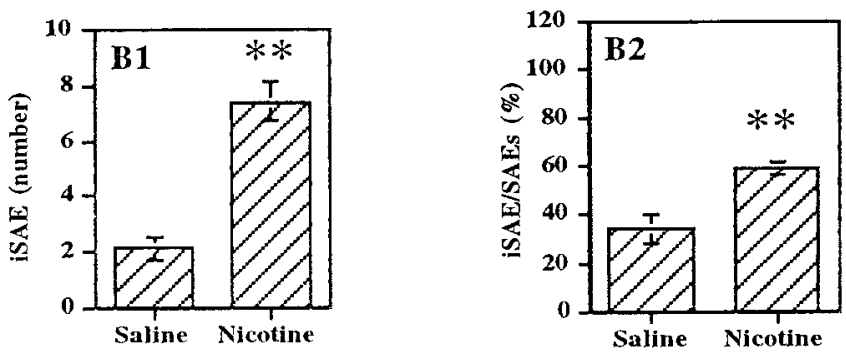

Later Maintenance
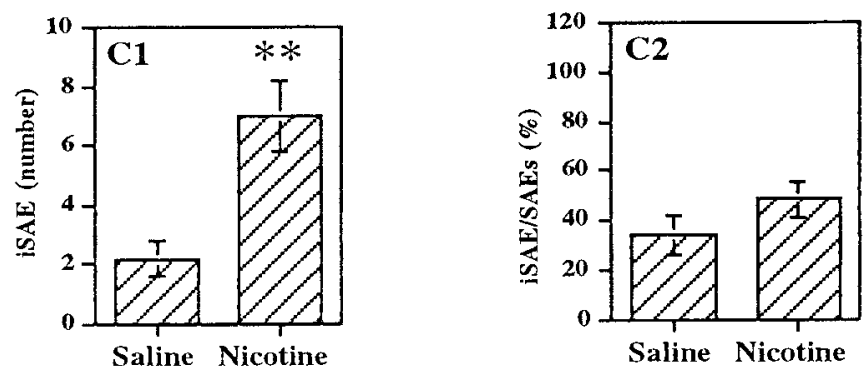

Figure 3. iSAEs increase during the three phases of self-administration. Nicotine SA resulted in an increase in both the mean number of iSAEs $(A 1, B 1, C 1)$ and the percentage of all SAEs associated with an increase in NE secretion (iSAEs/SAEs; $A 2, B 2$ ). ${ }^{* *} p<0.01$ compared with saline SA controls (for the nicotine SA groups, $n=6$ for acquisition, 12 for early maintenance, and 10 for late maintenance; for saline, $n=7$ ). The exception to this increased percentage during late maintenance $(C 2)$ may be indicative of the progression of partial desensitization to the effect of chronic nicotine SA (during acquisition, $t=3.99, p<0.01$ for iSAE; $t=$ $7.58, p<0.01$ for iSAE/SAEs; in maintenance, $F_{(3,31)}=10.47, p<0.01$ for iSAE; $F_{(3,31)}=4.77, p<0.01$ for iSAE/SAEs).

nance phase of nicotine SA was associated with a twofold increase in the mean number of SAEs $(p<0.01)$ (Table 1$)$. In addition, a fourfold increase in the number of iSAEs was observed $\left(F_{(3,31)}=10.47 ; p<0.01\right)$ (Fig. 3B1). The percentage of all SAEs associated with an $\mathrm{NE}$ increment (iSAE/total SAEs) also was higher in rats receiving nicotine $\left(F_{(3,31)}=4.77\right.$; $p<0.01$ ) (Fig. 3B2).

Figure $6 A$ shows that the mean NE increment associated with all iSAEs during the $9 \mathrm{hr}$ dialysis session was significantly higher in rats self-administering nicotine $\left(F_{(3 ., 31)}=3.57 ; p<0.05\right)$. The mean NE increment of the very first iSAE also was greater in nicotine than saline rats $\left(F_{(3,31)}=2.60 ; p<0.05\right)$ (Fig. 6B), whereas the mean NE increment of the last iSAE was not different $\left(F_{(3,28)}=0.75 ; p>0.05\right)$ (Fig. $\left.6 C\right)$. Comparison within the group receiving nicotine (Fig. $6 B, C$ ) also indicated that the $\mathrm{NE}$ increment of the first iSAE was significantly higher than the last in the same animals $(t=2.32 ; p<0.05)$. Figure $6 D$ demonstrates that the overall mean level of $\mathrm{NE}$ was twofold greater in rats self-administering nicotine compared with those receiving saline $\left(F_{(3,31)}=2.97 ; p<0.05\right)$. Thus, during the early maintenance phase, nicotine SA was associated with a significant increase in both the number of iSAEs and the NE increments achieved during these episodes. These NE increments were associated with early, but not late-occurring, iSAEs. Thus, despite the decline in the amount of $\mathrm{NE}$ secreted during late-occurring iSAEs, the overall level of NE present in the microdialysates obtained throughout the session was significantly elevated in the nicotine SA group.

The numbers of active and inactive lever presses were compared during the $23 \mathrm{hr}$ SA session in which early maintenance microdialysis was performed. The following number of active versus inactive lever presses occurred: $50.9 \pm 4.8$ active lever presses versus $31.5 \pm 5.7$ inactive lever presses in the nicotine SA group $(t=2.95 ; p<0.01 ; n=12)$; and $27.0 \pm 6.7$ active lever presses versus $31.7 \pm 12.6$ inactive lever presses in the saline group $(t=1.62 ; p>0.05 ; n=7)$. Thus, active lever presses exceeded inactive presses only in the nicotine SA group.

\section{PVN NE secretion and SAEs during the later maintenance phase}

By the time microdialysis was performed in the later phase of maintenance, animals had been self-administering nicotine for $18.6 \pm 0.8 \mathrm{~d}$ (range of 14-25 d). Figure 7 shows the SAEs and PVN NE levels in representative animals receiving nicotine $(A$; $n=10)$ or saline $(B ; n=6)$ in response to active lever presses. Comparison of the two indicates that total SAEs continued to remain elevated in the animal self-administering nicotine. This also was apparent in the group data shown in Table 1. Similar to the observations made during the early maintenance phase, the mean number of total injections, as well as SAEs, were significantly greater in nicotine-treated rats. Furthermore, Figure $3 C$ shows that the number of iSAEs continued to be significantly greater in the nicotine rats $\left(F_{(3,31)}=10.47 ; p<0.01\right)(C 1)$, although the percentage of iSAEs/total SAEs was not different $(C 2)$. Thus, in late maintenance, the number of SAEs and iSAEs remained elevated in nicotine SA animals.

Similar to the NE responsiveness noted during the early maintenance phase, Figure $8 A$ shows that the mean NE increment of all iSAEs was greater in the nicotine group during later maintenance $\left(F_{(3,31)}=3.57 ; p<0.05\right)$. In addition, the average $\mathrm{NE}$ increment of the first, but not the last, iSAE was significantly greater in the nicotine group [Fig. $8 B\left(F_{(3,31)}=2.60 ; p<0.05\right)$, $C\left(F_{(3,28)}=0.75 ; p>0.05\right]$. The overall mean NE level in the nicotine group was $79 \%$ higher than in saline rats $\left(F_{(3,31)}=2.97\right.$; $p<0.05$ ) (Fig. 8D). Comparing the average NE increment of all iSAEs during early versus later maintenance (Figs. 6 $A$, $8 A$, respectively), there was a considerable reduction in NE responsiveness by the later phase. Thus, PVN NE secretion was enhanced by nicotine SA during later maintenance, although to a lesser extent than in early maintenance.

The numbers of active and inactive lever presses were compared during the $23 \mathrm{hr}$ SA session in which later maintenance microdialysis was performed. The following number of active versus inactive lever presses occurred: $45.2 \pm 7.3$ active lever presses versus $29.7 \pm 5.9$ inactive lever presses in the nicotine SA group $(t=2.08 ; p<0.05 ; n=10)$; and $22.3 \pm 5.0$ active lever presses versus $29.2 \pm 10.5$ inactive lever presses in the saline 


\begin{tabular}{|c|c|c|c|c|}
\hline & \multicolumn{2}{|l|}{ Nicotine } & \multicolumn{2}{|l|}{ Saline } \\
\hline & Injections & SAEs & Injections & SAEs \\
\hline Acquisition (day 1) & $9.5 \pm 8.6$ & $5.0 \pm 1.7$ & $11.8 \pm 7.8$ & $6.0 \pm 1.8$ \\
\hline Early maintenance ${ }^{\#}$ & $31.5 \pm 3.6^{*}$ & $12.6 \pm 1.2^{* *}$ & $15.7 \pm 5.1$ & $6.7 \pm 0.8$ \\
\hline Later maintenance ${ }^{\#}$ & $31.5 \pm 6.1^{*}$ & $14.9 \pm 1.7^{* *}$ & $13.8 \pm 3.7$ & $6.5 \pm 1.1$ \\
\hline
\end{tabular}

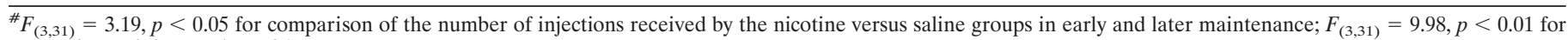
comparison of the number of SAEs.

${ }^{*} p<0.05$ or ${ }^{* *} p<0.01$ for post hoc comparisons with saline.
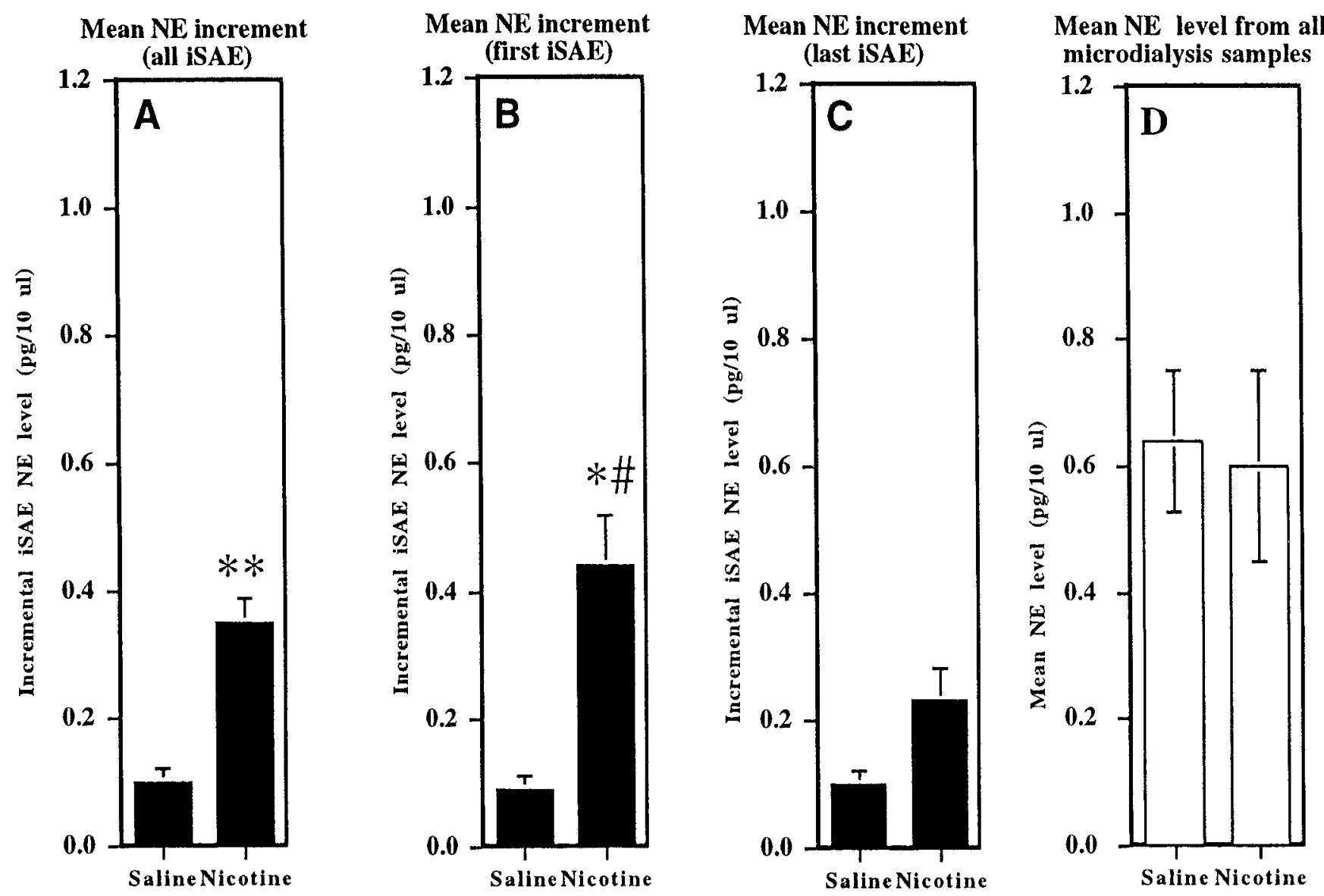

Figure 4. NE peak incremental response: day 1 of the acquisition period. The mean incremental response of all iSAEs throughout the entire 9 hr dialysis session $(A)$ was 3.5-fold higher in rats self-administering nicotine $(t=5.39 ; p<0.01)$, as was the level of NE in response to the first nicotine injection(s) $(B ; t=2.69 ; p<0.025)$. However, there was no difference in the $\mathrm{NE}$ response after $9 \mathrm{hr}(C ; t=1.38 ; p>0.05)$, indicating a decline in $\mathrm{NE}$ response over time. In addition, the overall mean NE levels, calculated from all of the samples collected during the 9 hr microdialysis session, were similar in both the nicotine and saline groups $(D ; t=0.57 ; p>0.05) . n=6$ in each group. ${ }^{*} p<0.05,{ }^{* *} p<0.01$ compared with saline control; $\# p<0.05$ compared with the last iSAE peak.

group ( $t=0.61 ; p>0.05 ; n=6)$. Thus, active lever presses exceeded inactive presses only in the nicotine SA group.

\section{Differences in intra-day PVN NE secretion during early and late-occurring iSAEs}

The foregoing data analyses showed that NE responsiveness to nicotine SA declined throughout the microdialysis session in all stages of SA. To clarify this, several potential factors were evaluated. First, to test whether the efficiency of the probe decreased during the $9 \mathrm{hr}$ microdialysis period, the in vitro $\mathrm{NE}$ recovery rate was measured in six microdialysis probes both before and after 9 hr of in vivo microdialysis. Microdialysis probe efficiency did not change during the $9 \mathrm{hr}$ session: $5.7 \pm 0.4 \%$ before microdialysis,
$5.2 \pm 0.5 \%$ after $(t=0.79 ; p>0.05)$. Second, to determine whether the reduced $\mathrm{NE}$ response associated with late-occurring iSAEs was specific to nicotine SA or attributable to the depletion of axonal $\mathrm{NE}$, yohimbine $(6 \mathrm{mg} / \mathrm{kg}$, i.p.) was administered during early maintenance nicotine SA, $8 \mathrm{hr}$ after the beginning of a microdialysis session. Figure 9 shows that peak NE release occurred 21-28 min after yohimbine, and no difference was seen between the two groups $\left(F_{(13,102)}=0.94 ; p>0.05 ; n=4\right)$. Thus, the secretory pool of PVN NE was not depleted during nicotine SA.

Third, to determine whether the intra-day differences in PVN $\mathrm{NE}$ secretion reflect reduced responsiveness to nicotine, groups 

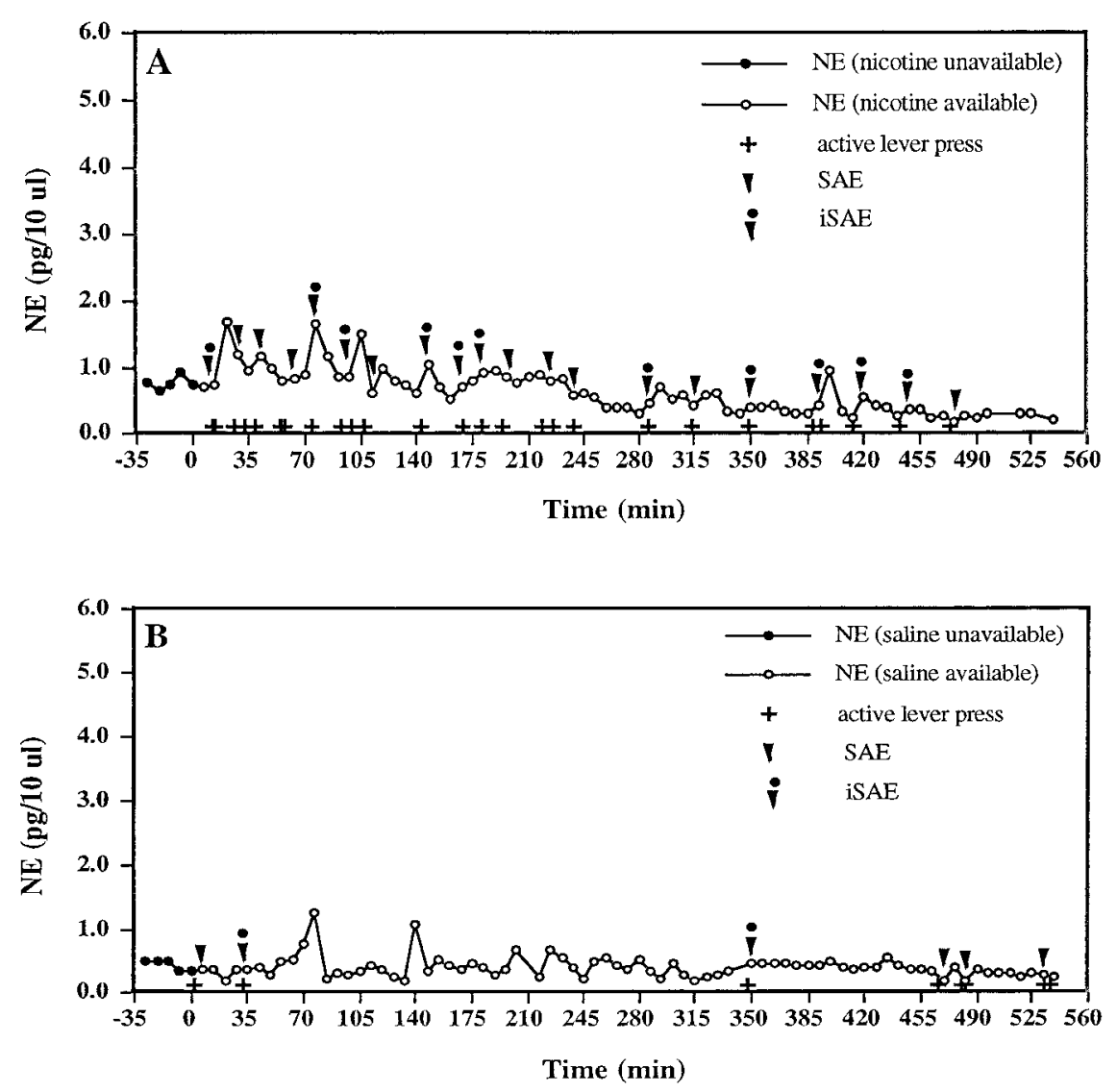

Figure 5. SAEs and NE levels in PVN microdialysates obtained during the early maintenance period. As in Figure 1, SAEs and NE levels are shown for two representative rats, nicotine SA $(A)$ and saline SA $(B)$. These rats were dialyzed $\sim 9.2 \pm 0.6 \mathrm{~d}$ after nicotine or saline initially became available for SA. In contrast to acquisition, early maintenance was associated with a twofold increase in the mean number of SAEs and a fourfold increase in iSAEs.
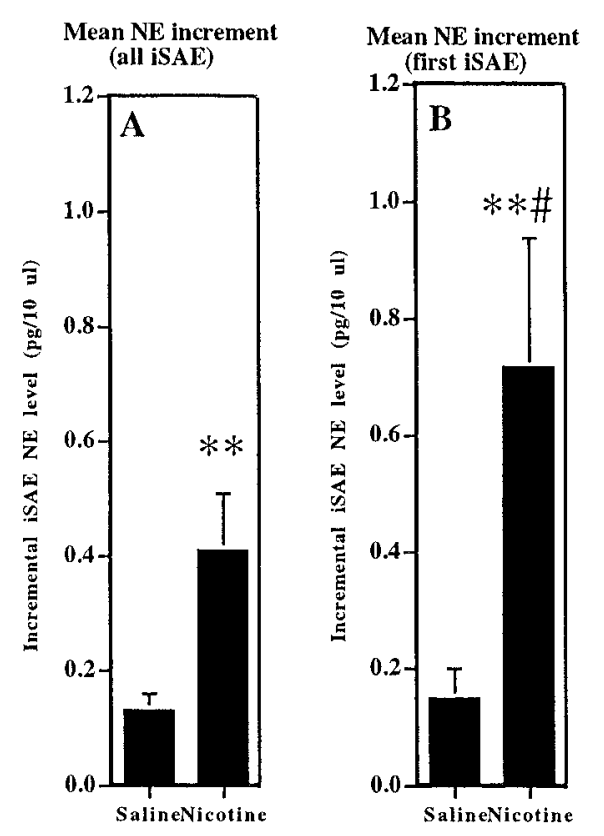

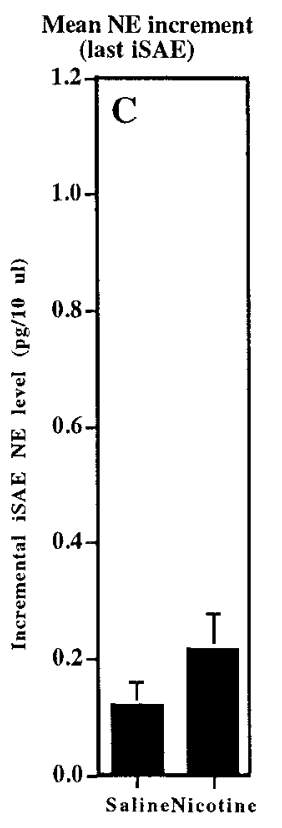

self-administering nicotine during the early maintenance phase or receiving saline were each given two consecutive passive injections of nicotine in the morning and then again $7 \mathrm{hr}$ thereafter. Figure 10 shows the incremental NE response to passively administered injections of nicotine in rats that had been receiving saline $(A)$ versus nicotine $(B)$ when they pressed the active lever. In the saline group, the variance in the incremental response to

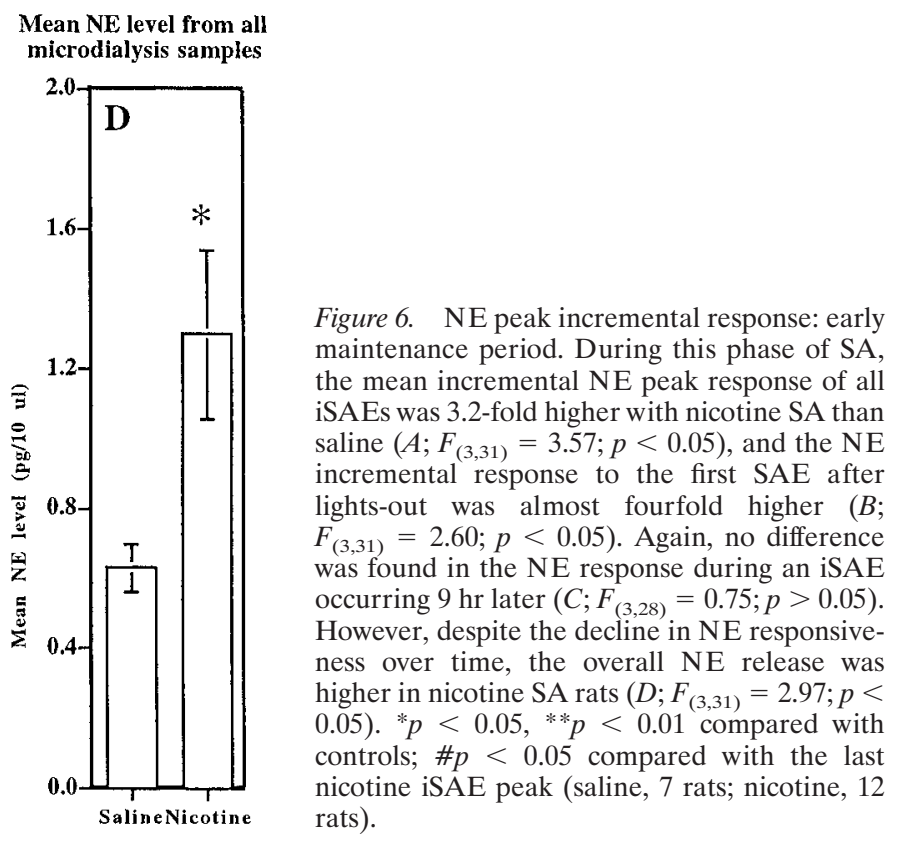

the first injection of nicotine was attributable to a single animal that, for unknown reasons, had both a higher NE baseline and response. In both groups, the NE response to the initial pair of passive nicotine injections was significantly greater than to the second set $(t=4.03, p<0.01$ for saline; $t=3.05, p<0.05$ for nicotine; $n=4$ each group). These findings indicate that reduced $\mathrm{NE}$ responsiveness to repeated treatment with passive nicotine 
Figure 7. SAEs and NE levels in PVN microdialysates obtained during the late maintenance period. SAEs and NE secretion in two representative rats during a $9 \mathrm{hr}$ microdialysis session (described in Fig. 2) performed $\sim 18.6 \pm 0.8 \mathrm{~d}$ after nicotine or saline initially became available for SA: nicotine SA $(A)$ and saline SA $(B)$. The total number of both SAEs and ISAEs remained elevated with nicotine SA.
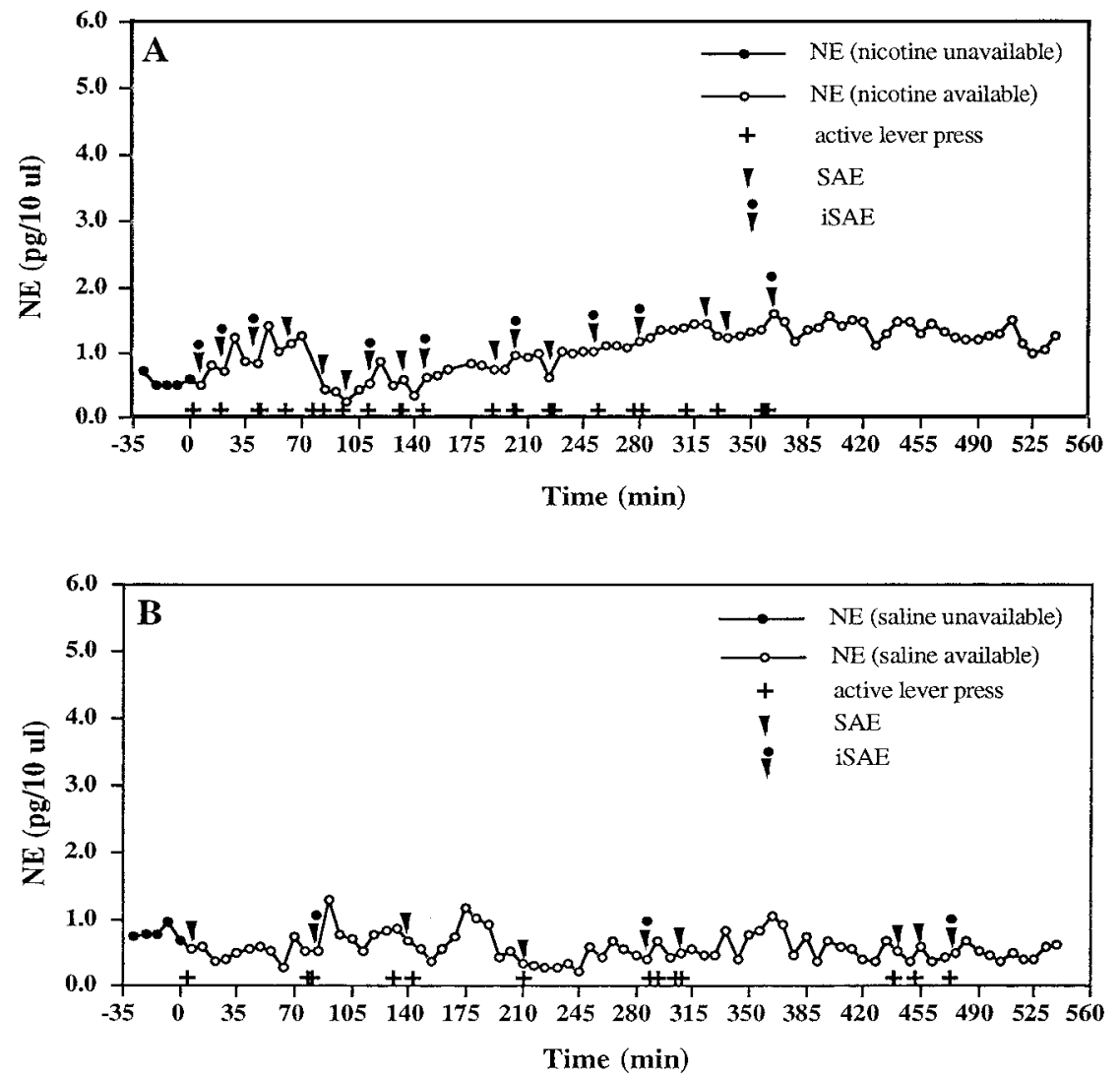

Figure 8. NE peak incremental response: late maintenance period. The average increment of all iSAEs over the $9 \mathrm{hr}$ microdialysis session was still higher with nicotine SA than saline SA $(A$; $\left.F_{(3,31)}=3.57 ; p<0.05\right)$ during this stage, as was the NE response to the first SAE after lights out $\left.\left(B ; F_{(3,31)}=2.60 ; p<0.05\right)\right)$. Although NE levels showed an overall increase of $79 \%\left(D ; F_{(3,31)}=\right.$ 2.97; $p<0.05)$, the decline in NE responsiveness during an iSAE $9 \mathrm{hr}$ later was still apparent $(C)$. ${ }^{*} p<0.05$ compared with controls (saline, 6 rats; nicotine, 10 rats).

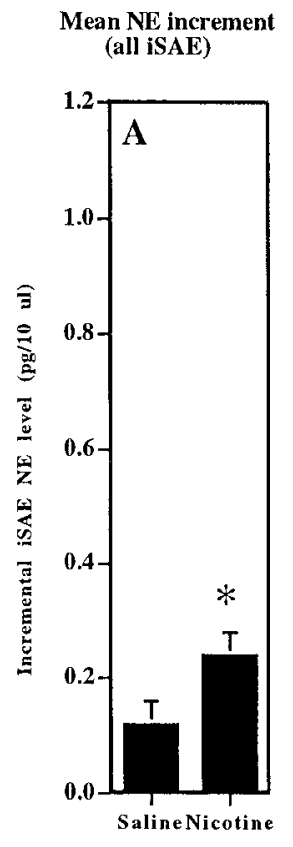

occurred in both groups, even when nicotine was administered as long as $7 \mathrm{hr}$ apart. These experiments also showed that the incremental $\mathrm{NE}$ response to the first set of passive nicotine injections was sixfold greater in the saline group, previously naïve to nicotine compared with the nicotine SA group $(t=3.941 ; p<$ $0.05)$. This suggests that previous chronic exposure to selfadministered nicotine reduced the NE responsiveness to passive
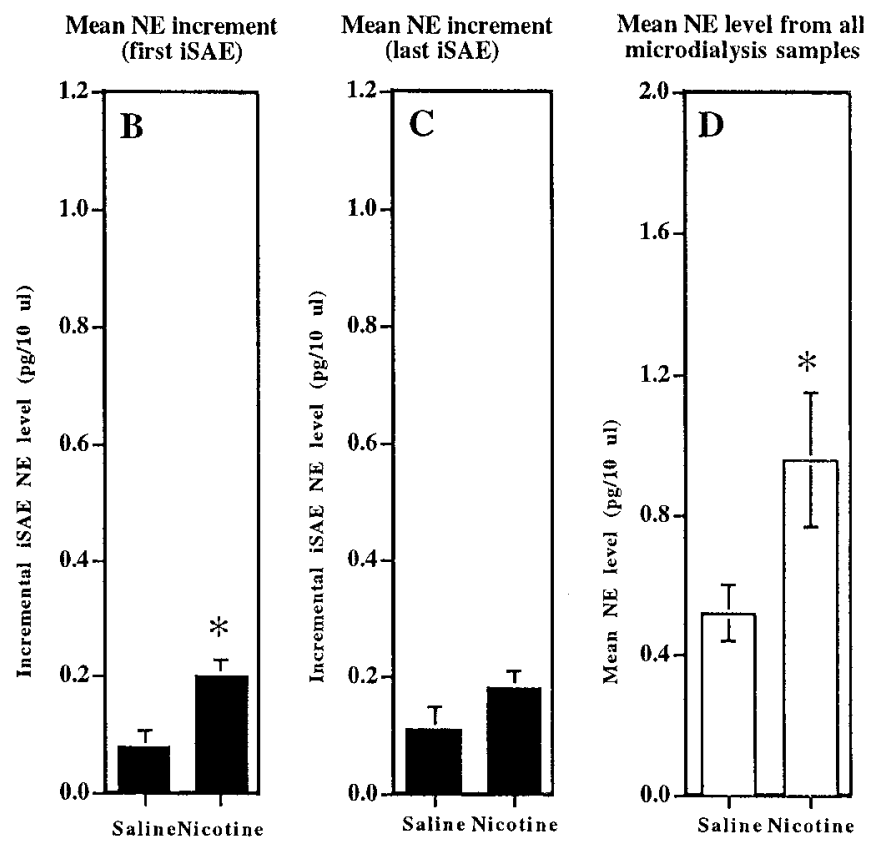

delivery of the drug, even when administered early in the microdialysis session.

Fourth, to determine whether there was a diurnal influence on $\mathrm{NE}$ responsiveness to nicotine that might have an impact on the intra-day differences observed, two groups of animals that received saline after pressing the "active" lever were each tested by receiving two consecutive passive injections of nicotine at only 


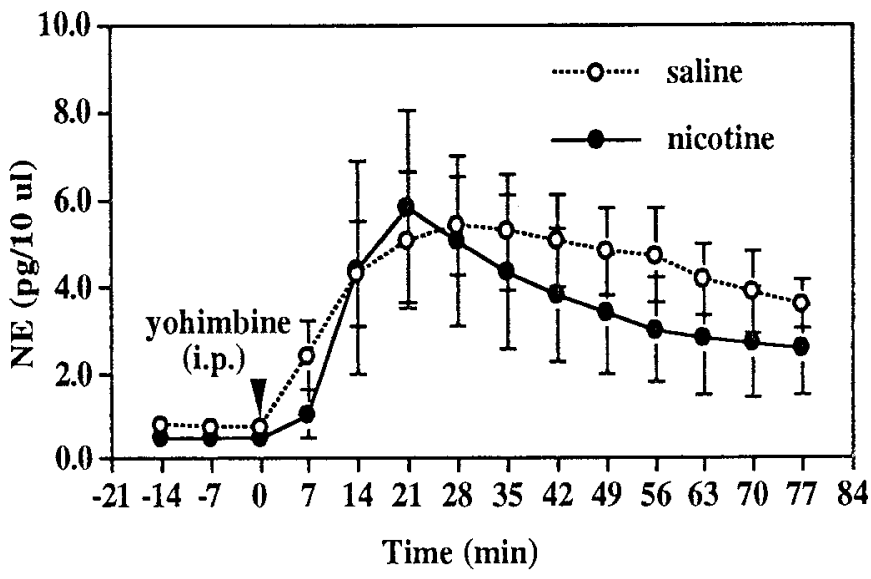

Figure 9. Effect of yohimbine on NE release in self-administering rats. To determine whether a depletion of axonal stores might underlie the reduction in NE release at the end of a $9 \mathrm{hr}$ dialysis session (see $C$ in Figs. $4,6,8), 6 \mathrm{mg} / \mathrm{kg}$ yohimbine was administered intraperitoneally $8 \mathrm{hr}$ into the session (at $0 \mathrm{~min}$ ) and dialysis continued. NE levels in nicotine SA rats were comparable with those in saline controls, demonstrating that the secretory pools were comparable $\left(F_{(13,102)}=0.94 ; p>0.05\right)$. Therefore, the reduction in NE response was specific to nicotine SA. $n=4$ per group.

one of the two time points discussed above $(0$ or $+7 \mathrm{hr})$. No differences were observed in the NE released by two consecutive nicotine injections, whether delivered at lights off $(0 \mathrm{hr})$ or $7 \mathrm{hr}$ thereafter [NE increment (picograms per $10 \mu \mathrm{l}$ ): $1.6 \pm 0.3$ or $1.1 \pm 0.2$, respectively; $t=0.832 ; p>0.05 ; n=5$ ].

Last, to assess whether nicotinic acetylcholine receptors (nAChRs) may have resensitized during the nicotine-free interval that separated the last nicotine injection in the previous SA session from the first injection on the day of microdialysis, the following were calculated: (1) the mean duration of this time period; and (2) the linear regression between the duration of the nicotine-free interval and the peak NE response to the first iSAE in individual rats. The following nicotine-free intervals were found: during early maintenance, $256.6 \pm 65.6 \mathrm{~min}$; during late maintenance, $267.8 \pm 69.5 \mathrm{~min}$. These prolonged nicotine-free intervals suggest that resensitization may have occurred followed by subsequent desensitization during the microdialysis session (induced by frequent nicotine injections). However, the linear regression analysis between the nicotine-free interval and the peak NE response failed to show significance (early maintenance, $r=0.40, p=0.19, n=12$; later maintenance, $r=0.05, p=0.69$, $n=10)$. This analysis indicates that the extent of this nicotinefree time interval was not a significant factor in determining the magnitude of the NE response to the first iSAE.

\section{DISCUSSION}

During early acquisition or the early and later maintenance phases of nicotine SA, rats had a significantly greater number of iSAEs, as well as a higher average NE increment in all iSAEs, compared with saline animals. These results demonstrate that PVN NE secretion is induced in coordination with the selfadministration of nicotine $(0.03 \mathrm{mg} / \mathrm{kg}$, i.v.). These findings, along with data showing that the overall NE level was higher in rats during the early and later maintenance phases of nicotine SA, indicate that nicotine SA stimulated the secretion of PVN NE. Comparisons of early to later maintenance also indicated that lesser amounts of PVN NE were secreted in response to nicotine SA during later maintenance. Moreover, nicotine SA was associ-
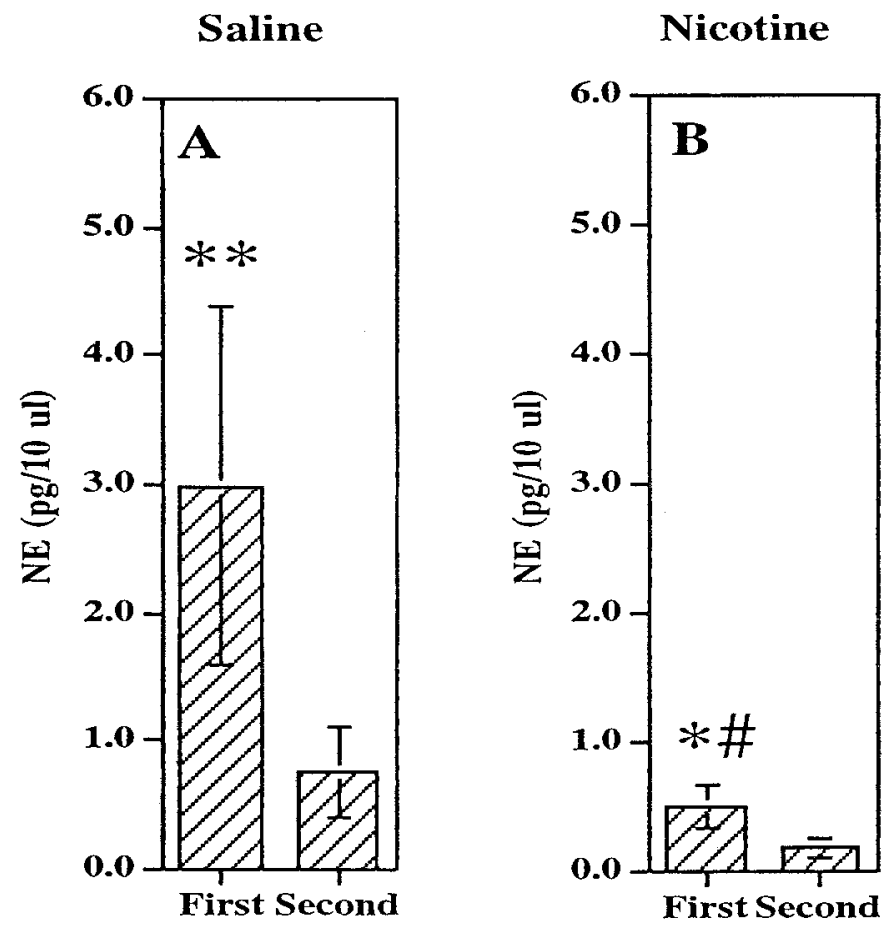

Figure 10. Effect of passively administered nicotine on NE release in self-administering rats. To determine whether the reduced NE responsiveness to nicotine could be attributable to diurnal differences, rats in the early maintenance phase of SA were given two consecutive nicotine injections at lights out $(0 \mathrm{~min})$ and then again $7 \mathrm{hr}$ later. In both the saline SA cohort $(A)$ and those self-administering nicotine $(B)$, the NE responses to the second set of injections passively administered $7 \mathrm{hr}$ after the first were significantly lower, indicating a prolonged reduction in both groups. In addition, the NE response to the first, passively delivered injections was significantly lower in rats with previous chronic exposure via nicotine SA. ${ }^{*} p<0.05$ or ${ }^{* *} p<0.01$ compared with the second set of injections; $\# p<0.05$ compared with saline SA rats receiving the first set of injections. $n=4$ per group.

ated with diminishing NE responsiveness within each daily SA session, regardless of the phase of SA. Therefore, a dose of 0.03 $\mathrm{mg} / \mathrm{kg}$ nicotine stimulates sustained PVN NE secretion in rats self-administering the drug, although the magnitude of the response declines both within each daily session and with the duration of SA. To our knowledge, this is the first report on CNS $\mathrm{NE}$ secretion in rats self-administering any drug and the first observation of neurotransmitter secretion in animals with unlimited access to self-administered nicotine.

The PVN NE secretory response to nicotine SA may depend on activation of nAChRs located in the brainstem. This is supported by data showing that mecamylamine, an nAChR antagonist, blocked intravenously administered nicotine-induced NE secretion in the PVN when the antagonist was microinjected into the fourth ventricle (Fu et al., 1997). The nucleus tractus solitarius (NTS) has been identified as the brainstem site containing the noradrenergic neurons that mediate nicotine-stimulated PVN $\mathrm{NE}$ secretion. Within the A2 and C2 regions of the NTS, intravenously administered nicotine stimulated c-fos expression in neurons coexpressing tyrosine hydroxylase, the rate-limiting enzyme in catecholamine biosynthesis. Activation of these NTS neurons also was blocked by administering mecamylamine into the fourth ventricle (Valentine et al., 1996). Comparing locus ceruleus and NTS, higher concentrations of systemic nicotine were required to activate locus ceruleus neuron c-fos expression 
(Matta et al., 1993a). In addition, the $\mathrm{ED}_{50}$ dose required to stimulate $\mathrm{ACTH}$ secretion was significantly higher for nicotine microinjected into locus ceruleus than NTS. Therefore, NTS noradrenergic inputs to $\mathrm{PVN}$ are more sensitive to nicotinic stimulation (Matta et al., 1993b); nonetheless, locus ceruleus noradrenergic afferents may contribute to the NE in PVN microdialysates.

Presynaptic nAChRs have been shown to mediate NE release in certain brain regions. For example, studies with hippocampal synaptosomes and slices have shown NE secretion induced by nicotinic agonists and blocked by mecamylamine (Sacaan et al., 1995; Clarke and Reuben, 1996). However, the effect of nicotine SA on PVN NE release is unlikely to depend on presynaptic nAChRs on NE terminals within the PVN, because microinjection of mecamylamine into the PVN was completely ineffective in blocking the release of NE (Fu et al., 1997) or ACTH (Matta et al., 1990) by intravenously administered nicotine.

In all three phases of nicotine SA (as shown in Figs. 4, 6, 8), the $\mathrm{NE}$ increment of the last iSAE was not significantly greater in nicotine SA compared with saline animals. Thus, a reduction in the NE responsiveness to nicotine SA was consistently observed within each day; this was independent of the number of days that nicotine was self-administered. Loss of efficiency of the microdialysis probe did not account for these observations. Furthermore, the reduced NE response was specific to nicotine SA, because yohimbine-induced NE secretion was unaffected when tested toward the end of a microdialysis session. Thus, nicotine SA did not deplete the secretory pools of axonal NE.

The fact that previous exposure to nicotine results in reduced responsiveness to the biochemical or behavioral effects of subsequent treatment(s) has long been thought to be mediated through receptor desensitization (Marks et al., 1985; Rapier et al., 1988; Rowell and Hillebrand, 1994). Indeed, microdialysis studies have shown a rapid reduction in both PVN and hippocampal NE secretion after a single passive injection of nicotine (Sharp and Matta, 1993; Fu et al., 1998). However, once reduced, repeated nicotine administration at constant $100 \mathrm{~min}$ intervals resulted in similar NE responses to the second, third, and fourth injections. This suggests that the reduction in $\mathrm{NE}$ responsiveness to passive administration occurs rapidly but does not proceed to completion.

The pattern of reduced PVN NE secretion was different in nicotine SA rats compared with the aforementioned studies on animals receiving drug passively. In early maintenance, the first two iSAEs that occurred during the microdialysis period were separated by $82.4 \pm 16.7 \mathrm{~min}$, yet the $\mathrm{NE}$ response to the second was only $18 \%$ less than the first $(0.72 \pm 0.22 \mathrm{pg} / 10 \mu \mathrm{l}$ for the first iSAE and $0.59 \pm 0.15 \mathrm{pg} / 10 \mu \mathrm{l}$ for the second). Furthermore, in later maintenance, $\mathrm{NE}$ secretion did not differ between the first two iSAEs $(0.20 \pm 0.03 \mathrm{pg} / 10 \mu \mathrm{l}$ for the first iSAE and $0.23 \pm 0.05$ $\mathrm{pg} / 10 \mu \mathrm{l}$ for the second), although these two episodes were $103.5 \pm 35.6 \mathrm{~min}$ apart. In contrast, we previously reported, using nicotine-naïve animals, that the second PVN NE response was $\sim 50 \%$ of the first when passive nicotine injections were separated by 100 min (Sharp and Matta, 1993). This difference in the NE response to self-administered versus passive delivery of nicotine may be attributable to the fact that at least partial desensitization had already taken place by early maintenance. This is supported by the results of the experiment wherein rats were given two sets of paired passive injections. The NE response in animals that were previously self-administering nicotine was sixfold less than in animals that were naïve to nicotine (Fig. 10), probably reflect- ing a preexisting reduction in NE responsiveness to the neuropharmacological effects of nicotine.

PVN NE responsiveness appeared to decline further during a $9 \mathrm{hr}$ microdialysis session in which the NE increment of the first iSAE was significantly greater than the last in animals receiving nicotine, for both the acquisition and early maintenance phases. Moreover, in all three phases, the NE increment was only greater during the first iSAE, but not the last, in rats receiving nicotine compared with saline. This probably reflects a greater degree of desensitization that develops during the lights-off phase of the daily light cycle when the animals administered nearly $80 \%$ of their daily nicotine. In addition to desensitization, it is likely that some of the difference between the NE response to the first versus the last iSAE reflects resensitization of $\mathrm{nAChRs}$ resulting in increased responsiveness to the first iSAE. Although the linear regression analysis did not provide evidence of resensitization during the 4-4.5 hr nicotine-free interval (from the last injection in the previous SA session to first injection during the microdialysis session), resensitization may have taken place during the first $8 \mathrm{hr}$ of the preceding "lights on" interval (inactive phase) when only $\sim 20 \%$ of the daily nicotine dose was delivered. During this time period, blood nicotine levels may drop sufficiently because of the greatly reduced frequency of nicotine delivery to permit resensitization. In addition to resensitization, it is possible that nicotine may have become a novel stimulus by the time that the first iSAE occurred. This might occur if some lever press activity persisted, despite the extinction of cue lights during the daily $1 \mathrm{hr}$ housekeeping interval when nicotine was unavailable. Thus, the delivery of nicotine during the early iSAEs of the subsequent SA session may have become unexpected, and such novelty may have facilitated NE responses.

By late maintenance, the number of iSAEs, the average NE peak height of iSAEs, and the increment in the first peak of the day, as well as the mean value over the $9 \mathrm{hr}$ microdialysis period, were still higher than saline rats. These results indicate that PVN NE continued to be released after nicotine SA at this stage. This is consistent with human cigarette smoking. When one cigarette was smoked, serum NE levels were elevated in 10 smokers after $1.5 \mathrm{hr}$ without cigarettes (Pomerleau, 1992). In addition, nicotine elevated plasma hormones (e.g., arginine vasopressin and $\beta$-endorphin) in smokers instructed to smoke after overnight deprivation (Pomerleau et al., 1983). Furthermore, the first cigarette of the day tended to induce greater responses (Parrott, 1994). This phenomenon also was observed in the present study: throughout all three phases of SA, NE levels were greater during the first iSAE in rats self-administering nicotine. These NE levels were greater than those present in the last iSAE during acquisition and early maintenance. Therefore, our nicotine SA model may reflect the CNS noradrenergic responses that occur with human cigarette smoking.

The present study is the first report on NE secretion during nicotine SA. Nicotine SAEs were significantly associated with increased PVN NE secretion during acquisition, early maintenance, and later maintenance. The PVN NE secretion in response to nicotine SA may be induced through nAChRs located in brainstem noradrenergic regions (especially the NTS). However, the NE response to nicotine SA declined during each microdialysis session and also by later maintenance. These changes are consistent with the progression of partial desensitization to the effect of nicotine SA on PVN NE secretion. 


\section{REFERENCES}

Benowitz NL, Jacob P (1984) Daily intake of nicotine during cigarette smoking. Clin Pharmacol Ther 35:499-504.

Clarke PBS, Reuben M (1996) Release of $\left[{ }^{3} \mathrm{H}\right]$-noradrenaline from rat hippocampal synaptosomes by nicotine: mediation by different nicotinic receptor subtypes from striatal $\left[{ }^{3} \mathrm{H}\right]$-dopamine release. Br J Pharmacol 117:595-606.

Fu Y, Matta SG, Valentine JD, Sharp BM (1997) Adrenocorticotropin response and nicotine-induced norepinephrine secretion in the rat paraventricular nucleus are mediated through brainstem receptors. Endocrinology 138:1935-1943.

Fu Y, Matta SG, Valentine JD, Sharp BM (1998) Desensitization and resensitization of norepinephrine release in the rat hippocampus with repeated nicotine administration. Neurosci Lett 241:147-150.

Mark GP, Hajnal A, Kinney AE, Keys AS (1999) Self-administration of cocaine increases the release of acetylcholine to a greater extent than response-independent cocaine in the nucleus accumbens of rats. Psychopharmacology 143:47-53.

Marks MJ, Stitzel JA, Collins AC (1985) Time course study of the effects of chronic nicotine infusion on drug response and brain receptors. J Pharmacol Exp Ther 235:619-628.

Matta SG, Singh J, Sharp BM (1990) Catecholamines mediate nicotineinduced adrenocorticotropin secretion via alpha-adrenergic receptors. Endocrinology 127:1646-1655.

Matta SG, Foster CA, Sharp BM (1993a) Nicotine stimulates the expression of cFos protein in the parvocellular paraventricular nucleus and brainstem catecholaminergic regions. Endocrinology 132:2149-2156.

Matta SG, Foster CA, Sharp BM (1993b) Selective administration of nicotine into catecholaminergic regions of rat brainstem stimulates adrenocorticotropin secretion. Endocrinology 133:2935-2942.

Matta SG, McCoy JG, Foster CA, Sharp BM (1995) Nicotinic agonists administered into the fourth ventricle stimulate norepinephrine secretion in the hypothalamic paraventricular nucleus: an in vivo microdialysis study. Neuroendocrinology 61:383-392.

Pacak K, Palkovits M, Kopin IJ, Goldstein DS (1995) Stress-induced norepinephrine release in the hypothalamic paraventricular nucleus and pituitary-adrenocortical and sympathoadrenal activity: in vivo microdialysis studies. Front Neuroendocrinol 16:89-150.

Palkovits M (1988) Distribution of neuropeptides in brain: A review of biochemical and immunohistochemical studies. In: Peptide hormones: effects and mechanisms of action, Vol 1, Neural distribution of brain peptides and changes during aging, Chap 1 (Negro-Vilar A, Conn PM, eds), pp 3-67. Boca Raton, FL: CRC.

Parrott AC (1994) Acute pharmacodynamic tolerance to the subjective effects of cigarette smoking. Psychopharmacology 116:93-97.
Paxinos G, Watson C (1986) The rat brain in stereotaxic coordinates, Ed 2. New York: Academic.

Pomerleau OF (1992) Nicotine and the central nervous system: biobehavioral effects of cigarette smoking. Am J Med 93:2S-7S.

Pomerleau OF, Fertig JB, Seyler LE, Jaffe J (1983) Neuroendocrine reactivity to nicotine in smokers. Psychopharmacology 81:61-67.

Rapier C, Lunt GG, Wonnacott S (1988) Stereoselective nicotineinduced release of dopamine from striatal synaptosomes: concentration dependence and repetitive stimulation. J Neurochem 50:1123-1130.

Rowell PP, Hillebrand JA (1994) Characterization of nicotine-induced desensitization of evoked dopamine release from rat striatal synaptosomes. J Neurochem 63:561-569.

Sacaan AI, Dunlop JL, Lloyd GK (1995) Pharmacological characterization of neuronal acetylcholine gated ion channel receptor-mediated hippocampal norepinephrine and striatal dopamine release from rat brain slices. J Pharmacol Exp Ther 274:224-230.

Sawchenko PE, Swanson LW (1982) The organization of noradrenergic pathways from the brain stem to the paraventricular and supraoptic nuclei in the rat. Brain Res Rev 4:272-325.

Schacht U, Leven M, Gerhards HJ, Hunt P, Raynaud JP (1982) Recent investigations on the mechanism of action of nomifensine. Int Pharmacopsychiatry 17:21-34.

Sharp BM, Matta SG (1993) Detection by in vivo microdialysis of nicotine-induced norepinephrine secretion from the hypothalamic paraventricular nucleus of freely moving rats: dose-dependency and desensitization. Endocrinology 133:11-19.

Summers KL, Giacobini E (1995) Effect of local and repeated systemic administration of $(-)$ nicotine on extracellular levels of acetylcholine, norepinephrine, dopamine, and serotonin in rat cortex. Neurochem Res 6:753-759.

Timmerman W, Westerink BH (1991) Importance of the calcium content inf used during microdialysis for the effects induced by D2 agonists on the release of dopamine in the striatum of the rat. Neurosci Lett 131:93-96.

Valentine JD, Matta SG, Sharp BM (1996) Nicotine-induced cFos expression in the hypothalamic paraventricular nucleus is dependent on brainstem effects: correlations with cFos in catecholaminergic and non-catecholaminergic neurons in the nucleus tractus solitarius. Endocrinology 137:622-630.

Valentine JD, Hokanson JS, Matta SG, Sharp BM (1997) Selfadministration in rats allowed unlimited access to nicotine. Psychopharmacology 133:300-304.

Wellman PJ (2000) Norepinephrine and the control of food intake. Nutrition 16:837-842.

Yoshihara T, Honma S, Honma K (1996) Prefeeding release of paraventricular neuropeptide $\mathrm{Y}$ is mediated by ascending noradrenergic neurons in rats. Am J Physiol 270:E596-E600. 